\title{
Heterogeneity of Plasma IRI Responses in Patients with IGT
}

Dear Sir,

Dr. Kosaka and Akanuma in their interesting letter raise questions of principle and of practice in respect of the suggested new criteria for diabetes mellitus (DM) and impaired glucose tolerance (IGT). It cannot be denied - and we have asserted it strongly ourselves - that IGT is a heterogeneous category in many respects, not least in the variety of mechanisms that we know to be responsible for the 'impaired' glucose response that defines it. Age, drugs, obesity, other diseases, early DM and other factors, alone or in combination, contribute to this category. We would certainly not regard it as a 'disease entity' in any sense, more a zone of diagnostic uncertainty or statistically increased risk in respect of the later development of DM (by the suggested new criteria). Indeed, we suggest that DM itself is heterogeneous in the sense that many mechanisms may give rise to the sustained and unequivocal hyperglycaemia which defines it.

Our suggestions are meant strictly to meet an operational clinical need - having found a certain degree of glucose intolerance, what should be done about it. Our Japanese colleagues are doing what we respectfully suggest is absolutely the correct thing, that is, trying to break the category of IGT down into its subgroups and define one in which the risk of worsening to diabetes is very high. They claim, for example, that a high proportion of their IGT subjects have low insulin responses and that it is from among those that the few 'worsening to diabetes' have emerged. This is a most valuable observation, though we must caution that one should not perhaps generalise too readily from it. They were able to report on progression in only 71 of 243 'starters' and we do not know how these were selected.

Much may depend also on how a 'population' of IGT is originally assembled in predicting its ultimate fate. A group of IGT assembled, for instance, from a population of relatives of diabetics or from women coming to light in antenatal clinics or referred to a clinic for some associated (or separate) pathology might well be differently constituted and subject as a group to a different metabolic fate than, for example the Bedford or Whitehall population of borderline diabetics which emerged from a population screening exercise. In neither of these latter groups did insulin measurement predict the 10 -year metabolic outcome.
Prior judgement of what represents a 'significant' IGT, perhaps involving some consideration of the accompanying insulin response, might thus bias the composition and subsequent natural history of a group. Ethnic differences themselves may play a role; characteristics of Japanese IGT may differ from British or Indian. Nevertheless there is a strong prima facie case for proposing, that, until it is shown to be untrue, in an ethnic group perhaps, or in some other specific situation, that those with the degree of glucose intolerance specified as IGT behave differently as a group from those specified as DM. It would be most valuable to be able to distinguish subsets of IGT whose metabolic fate could be more accurately predicted; and if this were established, there would be a strong case for making a special category for them. Insulin responses would seem to be an attractive analytical characteristic but the evidence that low responses are predictive is not compelling. In normal population samples, insulin responses cover a very wide range (and are moderately variable from time to time in the same person). This range and variability are to be expected as a background in any collection of people distinguished by IGT and must be taken into consideration.

It is very much to be hoped that research such as that of Professor Kosaka and his colleagues will in due course, bring more light into our current confusions and make it possible to describe with much greater precision the biochemical and endocrine basis of hyperglycaemia of all degrees, to predict its likely outcome and to indicate ways in which it may be improved. However, at present we cannot agree that the measurement of insulin plays a part in clinical diagnostic criteria for IGT or DM nor that knowledge of it has yet been shown to improve prediction as to outcome or to indicate more or less effective means of clinical management.

Yours sincerely,

H. Keen, MD, FRCP, Professor of Human Metabolism

Unit for Metabolic Medicine

Department of Medicine

Guy's Hospital Medical School

London Bridge

London

England 